\title{
Efecto letal agudo de los insecticidas en formulación comercial Imidacloprid, Spinosad y Thiocyclam hidrogenoxalato en obreras de Bombus atratus (Hymenoptera: Apidae)
}

\author{
Diego Riaño Jiménez \& José Ricardo Cure \\ Universidad Nueva Granada, Facultad de Ciencias Básicas y Aplicadas, Laboratorio de Biodiversidad y Ecología de \\ Abejas Silvestres, Bogotá - Colombia; diegorianojimenez@gmail.com; jose.cure@unimilitar.edu.co; \\ ecologiadeabejas@unimilitar.edu.co
}

Recibido 21-X-2015. Corregido 18-IV-2016. Aceptado 19-V-2016.

\begin{abstract}
Acute lethal effect of the commercial formulation of the insecticides Imidacloprid, Spinosad y Thiocyclam hidrogenoxalate in Bombus atratus (Hymenoptera: Apidae) workers. The effect of insecticides on bees has gained great attention, however, there are few studies that explore this issue on Neotropical bees. Bombus atratus is a neotropical species broadly distributed in Colombia and is considered an important pollinator of both Andean ecosystems and agroecosystems. However, as for many wild bees species, the effect of insecticides on $B$. atratus is unknow. In this study we determined the acute median lethal dose (LD50) of commercial formulations of insecticides Imidacloprid, Spinosad and Thiocyclam hydrogen oxalate, widely used in Colombia to control several pests of important crops. The LD50 was carried out by oral and contact routes, following and modifying the EPPO and OECD guidelines to perform LD50 on A. mellifera. We evaluated five doses for each route and insecticide, in a total of 25 medium-size workers for each dose by duplicate. Mortality was registered at 24, 48 and 72 hours after the experiment; and data were analyzed with the Probit regression model. For Imidacloprid, contacts and oral LD50 were $0.048 \mu \mathrm{g} / \mathrm{bee}$ and $0.010 \mu \mathrm{g} / \mathrm{bee}$, respectively. For Thiocyclam hydrogen oxalate, topical and oral LD50 were $0.244 \mu \mathrm{g} / \mathrm{bee}$ and $0.056 \mu \mathrm{g} / \mathrm{bee}$, respectively. For Spinosad, the oral LD50 corresponded to $0.28 \mu \mathrm{g} / \mathrm{bee}$; it was not possible to establish the LD50 for the contact route. The Hazard Quotient (HQ) and Index of Relative Toxicity indicated that all three active ingredients are highly toxic. We discussed the risk of the insecticides use on B. atratus, considering their chemical nature. Rev. Biol. Trop. 64 (4): 1737-1745. Epub 2016 December 01.
\end{abstract}

Key words: bumblebees, lethal median dose, DL50, pollinators, toxicity.

Las abejas son reconocidas por su importancia en los sistemas de producción agrícola debido a su actividad y eficiencia polinizadora (Greenleaf \& Kremen, 2006; Klein et al., 2009). Sin embargo, existe una alarma generalizada a nivel mundial debido a la reducción de las poblaciones de abejas silvestres y a la disminución de las colonias de abejas de la miel, lo que se atribuye, entre otras causas, al uso de los pesticidas agrícolas (Biesmeijer et al., 2006; Colla \& Packer, 2008; Rundlöf, Nilsson, \& Smith, 2008; Gordon, Rizzardi, Vinson, \& Griswold, 2009; Dar, Khan, \& Abass, 2010). A pesar de esto, son escasos los trabajos toxicológicos existentes a nivel letal y subletal, que muestran el efecto de dichos químicos en las diferentes abejas de las especies disponibles comercialmente (Van der Steen, 2001; Freitas et al., 2009; Pinheiro \& Freitas, 2010; Potts et al., 2010). La Dosis Letal media aguda (DL50) aunque es una prueba de laboratorio empleada principalmente para el registro de productos agroquímicos, también puede ser una herramienta para establecer el efecto de dichos productos en organismos benéficos para los cultivos, incluyendo a las abejas o incluso para establecer medidas para la mitigación de los efectos en las poblaciones silvestres, a partir 
de análisis derivados de la DL50, como lo es el Cociente de Riesgo (HQ por sus siglas en inglés) (Thompson \& Hunt, 1999; Thompson, 2001; Marrs \& Ballantyne, 2004). Por ejemplo, en Suramérica, Canadá, Estados Unidos y Europa, el registro de productos agroquímicos requiere tanto de pruebas toxicológicas, principalmente en Apis mellifera, como de los análisis derivados de estas (por ejemplo el Índice de Riesgo-HQ por sus siglas al inglés). A partir de esta información, se pueden hacen recomendaciones de su uso (toxicidad y las tasas de uso) para disminuir el riesgo en las abejas (incluyendo las especies silvestres) (Mineau et al., 2008). Sin embargo, varios estudios realizados en especies de abejas disponibles comercialmente y diferentes de $A$. mellifera (B. terrestris, B. occidentalis, B. lucorum, B. lapidarius, Megachile rotundata y Osmia lignaria) han demostrado que la sensibilidad a los insecticidas puede variar dependiendo de la especie de abeja (Thompson, 2001, Scott-Dupree, Conroy, \& Harris, 2009).

En el Neotrópico, es escaso el conocimiento sobre el efecto de los productos agroquímicos en abejas, a pesar de la biodiversidad con la que cuenta (cerca de 15000 especies) y de la importancia del servicio ecosistémico que proveen (Freitas et al., 2009, Pinheiro \& Freitas, 2010). La creciente expansión agrícola de esta región y el uso de pesticidas resaltan la importancia de desarrollar trabajos que evidencien el efecto de los agroquímicos en las especies de abejas silvestres (Belloti et al., 1990; Pinheiro \& Freitas, 2010). B. atratus es un abejorro neotropical, de amplia distribución en Colombia, con un rango altitudinal entre los 500 y $3200 \mathrm{~m}$ de elevación (Ospina, Lievano, \& Nates-Parra, 1987; Abrahamovich, Diaz \& Morrone, 2002) y es considerado uno de los polinizadores más importantes de plantas en los ecosistemas y agroecosistemas alto andinos (Nates-Parra \& González, 2000; Almanza, 2007). Adicionalmente, se ha desarrollado un sistema de cría de colonias en condiciones de cautiverio, con propósitos de determinar su actividad y efecto polinizador en cultivos colombianos (Aldana et al., 2007; Cruz, Almanza \& Cure, 2007;
Pacateque, Cruz, Aguilar, \& Cure 2012; Riaño, Veloza, M., Cure, J. R., \& Almanza, Veloza, Cure, \& Almanza, 2014). A pesar de su importancia, aún se desconoce el efecto de los insecticidas, por lo que éste estudio determinó la dosis letal media aguda (DL50) por exposición tópica y oral, en obreras de $B$. atratus, de las formulaciones comerciales a base de Imidacloprid, Spinosad y Thiocylam hidrogenoxalato, seleccionados por su amplio uso en cultivos colombianos (tomate, flores de corte, algodón, entre otros) para el control de plagas (Bojacá, Gil, Casilimas, \& Schrevens, 2012).

\section{MATERIALES Y MÉTODOS}

Cría de colonias de $B$. atratus: Para el desarrollo del estudio se criaron 101 colonias de $B$. atratus bajo condiciones de cautiverio en el laboratorio Cría de Abejorros de la Universidad Nueva Granada (N 4'56'34.88'" - O $\left.74^{\circ} 00^{\prime} 38,02^{\prime \prime}\right)$, siguiendo el protocolo de cría desarrollado por el laboratorio Biodiversidad y Ecología de Abejas Silvestres - BEAS (Cruz et al., 2007; Pacateque et al., 2012, Riaño et al., 2014). Éste esquema favoreció la diversidad genética de las pruebas (USDA, 2009) y a la vez favoreció la supervivencia de las colonias.

Insecticidas y rutas de exposición evaluados en obreras de $\boldsymbol{B}$. atratus: Se evaluó la toxicidad letal media aguda (DL50) por exposición oral (alimento contaminado) y tópica (contacto directo con la solución) de las presentaciones comerciales disponibles en Colombia de los ingredientes activos (i.a.): Spinosad - Solución Concentrada $12 \%\left(\right.$ Trace $\left.^{\mathrm{r}}\right)$, Thiocyclam hidrogenoxalato - Polvo Soluble $50 \%$ Evisect $\left.^{\circledR}\right)$, e Imidacloprid - Solución Concentrada $35 \%$ (Confidor $\left.^{\circledR}\right)$.

\section{Determinación de límites de respuesta} de los insecticidas: $\mathrm{Al}$ desconocerse el efecto de los insecticidas evaluados en las obreras de $B$. atratus, fue necesario realizar pruebas preliminares para establecer las dosis con límite de respuesta $100 \%$ de mortalidad con la menor dosis posible, que posteriormente se utilizó 
para establecer la DL50 definitiva. Se evaluaron cinco dosis preliminares producto de diluciones seriadas (factor 1:10) partiendo de la dosis promedio recomendada por el fabricante para cada producto. En cada dosis se evaluaron 10 obreras de $B$. atratus de tamaño medio (4 $\pm 0.1 \mathrm{~mm}$ de ancho cefálico y $0.22 \pm 0.05 \mathrm{~g}$ ) provenientes de diferentes colonias. Tanto las pruebas preliminares como las pruebas definitivas DL50 correspondieron a adaptaciones metodológicas de las propuestas para Apis mellifera (OECD, 1998a; OECD, 1998b; van der Steen, 2001; Thompson, 2001), que se describen detalladamente a continuación.

DL50 por exposición tópica: Luego de establecer la dosis mínima con respuesta de mortalidad $100 \%$ de cada producto por exposición tópica, se establecieron cuatro dosis adicionales a partir de diluciones seriadas (factor 1:10), para determinar la DL50 por exposición tópica definitiva (Cuadro 1). Cada producto se disolvió en agua desionizada y se le adicionó coadyuvante no iónico (Inex-A (Cosmoagro) -1 mL/litro de agua), con el propósito de disminuir la tensión superficial y así facilitar la adherencia del producto a las obreras. Para cada concentración se evaluaron 25 obreras siguiendo el mismo criterio de selección descrito en la sección anterior (OECD, 1998a.; OECD, 1998b; van der Steen, 2001; Thompson, 2001). Una vez aisladas, cada obrera fue adormecida con $\mathrm{CO}_{2}$ por un periodo de 10 segundos. Seguidamente, a cada individuo se le aplicó $5 \mu \mathrm{L}$ de cada concentración en la parte ventral del tórax, entre el segundo y tercer par de patas con una micropipeta de 0.5-10 $\mu \mathrm{L}$ (BIOPETTE) (van der Steen, 2001; Thompson, 2001). Cada obrera de $B$. atratus fue individualizada en una caja de Petri de $100 \mathrm{~mm}$ de diámetro y ubicada inmediatamente después en un cuarto climatizado. Las obreras fueron alimentadas con solución de sacarosa y agua $(1: 1 \mathrm{p} / \mathrm{v})$ y polen ad libitum. Los controles de cada prueba correspondieron a la administración tópica de agua desionizada y agua desionizada con coadyuvante. La mortalidad se evaluó a las 24, 48 y 72 horas después de la aplicación. Cada prueba se realizó por duplicado (Thompson, 2001; van der Steen, 2001). Si la mortalidad del control superaba el $10 \%$, la prueba era repetida (Thompson, 2001). Los residuos tanto químicos como biológicos fueron depositados en contenedores herméticos y descartados acorde con los protocolos establecidos por la institución.

DL50 por exposición oral: Luego de establecer la dosis mínima con respuesta de mortalidad $100 \%$ de cada producto por exposición oral, se establecieron cuatro dosis adicionales a partir de diluciones seriadas (factor 1:10) para determinar la DL50 por exposición oral definitiva (Cuadro 1). Cada producto fue disuelto en solución azucarada (solución de agua y sacarosa $1: 1 \mathrm{v} / \mathrm{p}$ ). Por cada dosis, se evaluaron 25 obreras de tamaño medio procedentes de diferentes colonias, las cuales eran individualizadas en frascos de vidrio con ventilación,

CUADRO 1

Descripción de la dosis (/L de agua) y concentración (g i.a/L agua.) de los productos comerciales usados y las concentraciones evaluadas en DL50 por exposición tópica $(\mathrm{T})$ y oral $(\mathrm{O})$ en obreras de $B$. atratus

\begin{tabular}{|c|c|c|c|c|c|c|c|}
\hline \multirow{2}{*}{ i.a } & \multirow{2}{*}{ Dosis } & \multirow{2}{*}{ Concentración $^{+}$} & \multicolumn{5}{|c|}{ Concentraciones evaluadas (g i.a./L) DL50 } \\
\hline & & & 1 & 2 & 3 & 4 & 5 \\
\hline $\operatorname{Im}$ & $1 \mathrm{~mL}$ & 0.35 & $\begin{array}{c}\mathrm{T}: 0.035 \\
\mathrm{O}: 0.0035\end{array}$ & $\begin{array}{c}\mathrm{T}: 0.017 \\
\mathrm{O}: 0.002\end{array}$ & $\begin{array}{c}\mathrm{T}: 0.008 \\
\mathrm{O}: 0.001\end{array}$ & $\begin{array}{l}\mathrm{T}: 0.0042 \\
\mathrm{O}: 0.0005\end{array}$ & $\begin{array}{c}\mathrm{T}: 0.0021 \\
\mathrm{O}: 0.0001\end{array}$ \\
\hline Th & $500 \mathrm{mg}$ & 0.25 & $\begin{array}{l}\mathrm{T}: 0.25 \\
\mathrm{O}: 0.1\end{array}$ & $\begin{array}{l}\mathrm{T}: 0.125 \\
\mathrm{O}: 0.02\end{array}$ & $\begin{array}{l}\mathrm{T}: 0.062 \\
\mathrm{O}: 0.008\end{array}$ & $\begin{array}{l}\mathrm{T}: 0.031 \\
\mathrm{O}: 0.001\end{array}$ & $\begin{array}{c}\mathrm{T}: 0.006 \\
\mathrm{O}: 0.0002\end{array}$ \\
\hline $\mathrm{Sp}$ & $0.3 \mathrm{~mL}$ & 0.036 & $\begin{array}{c}\mathrm{T}: 0.06 \\
\mathrm{O}: 0.135\end{array}$ & $\begin{array}{c}\mathrm{T}: 0.03 \\
\mathrm{O}: 0.074\end{array}$ & $\begin{array}{l}\mathrm{T}: 0.015 \\
\mathrm{O}: 0.037\end{array}$ & $\begin{array}{c}\mathrm{T}: 0.0075 \\
\mathrm{O}: 0.015\end{array}$ & $\begin{array}{c}\mathrm{T}: 0.00037 \\
\mathrm{O}: 0.009\end{array}$ \\
\hline
\end{tabular}

+: Concentración de i.a. a partir de la dosis recomendada por el fabricante para el manejo de plagas. Im: Imidacloprid; Th: Thiocyclam Hidrogenoxalato; Sp: Spinosad. 
y mantenidas sin alimento durante tres horas en un cuarto climatizado (temperatura, luminosidad y humedad relativa) para disminuir el efecto del estrés en los individuos (van der Steen, 2001; Thompson, 2001). Posteriormente, a cada obrera se le ofreció individualmente $10 \mu \mathrm{L}$ de la solución en pequeños alimentadores de polipropileno de $\propto 0.5 \mathrm{~mm}$ ubicados dentro de una caja de Petri de $100 \mathrm{~mm}$. Cada obrera era observada para asegurar el consumo de la totalidad del contenido y que no tuviera contacto directo con la solución. Posteriormente, los individuos eran ubicados en el cuarto climatizado bajo condiciones de oscuridad. El control correspondió a obreras mantenidas bajo las mismas condiciones que las obreras tratadas descritas anteriormente y a las que se les ofreció solución azucarada sin tratar. Cada prueba se realizó por duplicado (Thompson, 2001; van der Steen, 2001). La mortalidad se evaluó a las 24, 48 y 72 horas después de la aplicación (Thompson, 2001; van der Steen, 2001). Si la mortalidad del control superaba el $10 \%$, la prueba era repetida (Thompson, 2001). Los residuos tanto químicos como biológicos fueron depositados en contenedores herméticos y descartados acorde con los protocolos establecidos por la institución.

Los datos de la DL50 definitiva por exposición tópica y oral fueron analizados con el modelo de regresión PROBIT con el programa estadístico S.A.S para Windows (v. 9.2) para estimar la dosis letal media (DL50) por exposición tópica y oral de los tres insecticidas evaluados (Gómez, 1999).
Se calcularon los intervalos de confianza al $95 \%$ de las réplicas realizadas en cada prueba de toxicidad a partir del procedimiento propuesto por Robertson et al. (2007).

Se realizó el cálculo del Índice de Toxicidad Relativa DL50 (ITxR) para cada insecticida y tipo de exposición a partir del procedimiento propuesto por Robertson et al. (2007), el cual permite establecer la susceptibilidad de una población a un insecticida, a partir de la comparación de su toxicidad (DL50) con la de un insecticida de referencia (insecticida con la toxicidad más baja). Se calculó el coeficiente de riesgo (HQ) para los tres insecticidas para las dos vías de exposición a partir de Thompson y Hunt (1999) y USDA (2009).

\section{RESULTADOS}

Toxicidad letal media aguda (DL50) por exposición tópica: Para el producto comercial a base del i.a. Spinosad no fue posible establecer la DL50 por exposición tópica, debido a que no se estableció el límite de respuesta de $100 \%$ de mortalidad (Cuadro 2).

En el caso de los i.a. Imidacloprid y Thiocyclam hidrogenoxalato, los resultados obtenidos con el cálculo de la DL50 por exposición tópica, muestran que estos son altamente tóxicos para las obreras de $B$. atratus a partir de los rangos propuestos por USDA (2009) (DL50 $<1 \mu \mathrm{g}$ i.a./abeja) (Cuadro 2). Las réplicas realizadas para establecer la DL50 por exposición tópica de los dos i.a. no mostraron diferencias significativas $(\mathrm{p}>0.05)$.

CUADRO 2

Dosis aguda letal media (DL50) de los i.a. Imidacloprid (Im), Thiocyclam hidrogenoxalato (Th) y Spinosad (Sp) por exposición tópica y oral en obreras de B. atratus

\begin{tabular}{|c|c|c|c|c|c|c|c|}
\hline i.a. & Exposición & $\mathrm{n}$ & Pendiente $( \pm$ ES) & $\begin{array}{c}\text { DL } 50-\mu g \text { i.a/abeja } \\
(\text { IC-95\%) }\end{array}$ & $\begin{array}{c}\text { DL } 95-\mu g \text { i.a/abeja } \\
(\text { IC-95\%) }\end{array}$ & $X^{2}$ & $\mathrm{P}$ \\
\hline \multirow[t]{2}{*}{$\operatorname{Im}$} & Oral & 250 & $3.31(0.38)$ & $0.01(0.0086-0.011)$ & $0.031(0.025-0.045)$ & 0.98 & 0.8 \\
\hline & Tópica & 250 & $2.45(0.26)$ & $0.051(0.042-0.065)$ & $0.20(0.150 .31)$ & 0.39 & 0.75 \\
\hline \multirow[t]{2}{*}{ Th } & Oral & 250 & $1.80(0.19)$ & $0.056(0.041-0.074)$ & $0.45(0.29-0.84)$ & 1.96 & 0.6 \\
\hline & Tópica & 250 & $2.84(0.34)$ & $0.24(0.20-0.28)$ & $0.92(0.71-1.36)$ & 0.48 & 0.92 \\
\hline $\mathrm{Sp}$ & Oral & 250 & $3.17(0.31)$ & $0.28(0.24-0.33)$ & $0.94(0.75-1.30)$ & 1.21 & 0.74 \\
\hline
\end{tabular}

n: número total de obreras evaluadas; ES: error estándar; IC: intervalo de confianza; x2: chi cuadrado. 
CUADRO 3

Índice de toxicidad relativa (ITxR) de los i.a. Imidacloprid. Thiocyclam hidrogenoxalato y Spinosad en obreras de B. atratus

\begin{tabular}{|c|c|c|}
\hline \multirow{2}{*}{ i.a. } & \multicolumn{2}{|c|}{ Índice de toxicidad relativa (IC-95 \%) } \\
\hline & Contacto & Oral \\
\hline Imidaclorpid & $52.62(51.25-54.1)$ & $274.14(273.5-274.7)$ \\
\hline Thiocyclam hidrogenoxalato & $*$ & $42.08(40.29-43.66)$ \\
\hline Spinosad & $\mathrm{nc}$ & $*$ \\
\hline
\end{tabular}

IC: intervalo de confianza; *: Insecticida de referencia: corresponde al insecticida menos tóxico (DL50) en cada una de las vías de exposición. nc: No calculado.

\section{CUADRO 4}

Cociente de Riesgo (HQ) para las obreras de B. atratus a partir de las presentaciones comerciales a base de los i.a. Imidacloprid. Thiocyclam hidrogenoxalato y Spinosad

\begin{tabular}{|c|c|c|c|c|c|}
\hline \multirow{2}{*}{ Producto comercial } & \multirow{2}{*}{ i.a. } & \multirow{2}{*}{$\begin{array}{l}\text { cantidad producto } \\
\text { comercial/ha }\end{array}$} & \multirow{2}{*}{ g i.a./ha } & \multicolumn{2}{|c|}{ HQ } \\
\hline & & & & tópico & oral \\
\hline Confidor $\AA 350 \mathrm{SC}$ & Imidacloprid & $400 \mathrm{~mL}$ & 140 & 2745.1 & 14000 \\
\hline Evisect $\mathrm{S} \ltimes \mathrm{SP}$ & Thiocyclam hidrogenoxalato & $500 \mathrm{~g}$ & 250 & 1041.6 & 4464.3 \\
\hline Tracer ${ }^{\circledR} 120 \mathrm{SC}$ & Spinosad & $200 \mathrm{~mL}$ & 24 & $\mathrm{nc}$ & 85.7 \\
\hline
\end{tabular}

nc: no calculado.

Toxicidad letal media (DL50) por exposición oral: Los valores de DL50 obtenidos por exposición oral muestran que los i.a. Imidacloprid, Thiocyclam hidrogenoxalato y Spinosad son altamente tóxicos para las obreras de $B$. atratus a partir de los rangos propuestos por USDA (2009) (DL50 < $1 \mu$ g i.a./abeja) (Cuadro 2). Las réplicas realizadas para establecer la DL50 por exposición oral de cada i.a. no mostraron diferencias significativas $(p>0.05)$. En ninguno de los controles de las pruebas de toxicidad letal media se presentó mortalidad.

Índice de toxicidad relativa: A partir de los resultados obtenidos con la DL50 por exposición tópica y oral de los tres i.a. el cálculo del índice de toxicidad relativa, el producto comercial a base del i.a. Imidacloprid muestra la mayor toxicidad entre los insecticidas evaluados en los dos modos de exposición (Cuadro 3).

Cociente de riesgo - HQ: A partir del cálculo del HQ para las obreras de $B$. atratus se detectó que el producto comercial a base del i.a. Imidacloprid es altamente tóxico en las dos vías de exposición evaluadas (HQ > 2500). El i.a. Thiocyclam hidrogenoxalato es altamente tóxico por exposición oral (HQ > 2500) y moderadamente tóxico por exposición tópica (HQ 50 - 2500). El i.a. Spinosad mostró una toxicidad baja-moderada por exposición oral (HQ 50 - 2500) (Cuadro 4).

\section{DISCUSIÓN}

Es claro que el uso (indiscriminado) de insecticidas puede tener un efecto negativo en organismos no blanco, incluyendo aquellos que son benéficos para los cultivos como las abejas (Desneux, Decourtye, \& Delpuech, 2007; Sánchez-Bayo 2012). En Colombia, no existían reportes que establecieran la toxicidad de los agroquímicos en las abejas silvestres, por lo que el presente trabajo quiso contribuir no solo a establecer los valores de la dosis letal media, sino también, a proponer estrategias de uso de estos productos, teniendo en cuenta las características propias de los i.a. y el índice de riesgo (HQ) a partir de las tasas recomendadas para Colombia. 
Los resultados obtenidos demuestran que los insecticidas evaluados son altamente tóxicos para $B$. atratus por exposición tópica y oral al igual que lo obtenido en otras especies tanto del género Bombus como de otros grupos de abejas (exposición oral Imidacloprid > Thiocyclam hidrogenoxalato $>$ Spinosad; exposición tópica Imidacloprid $>$ Thiocyclam hidrogenoxalato) (Thompson, 2001, Scott-Dupree et al., 2009).

La alta toxicidad letal media del Imidacloprid por exposición tópica y oral obtenida es similar a lo reportado anteriormente para $B$. terrestris $(0.04 \mu \mathrm{g}$ i.a./abeja y $0.01 \mu \mathrm{g}$ i.a./abeja respectivamente) (Marletto, Patetta, \& Manino, 2003). Los estudios sobre el efecto de las trazas traslocadas tanto al polen como al néctar, muestran un efecto subletal que afectan la reproducción y el comportamiento en $B$. terrestris y A. mellifera (Blacquière et al., 2012), sin embargo, éste aspecto no se ha establecido en B. atratus. En Colombia, existen cerca de 50 productos comerciales registrados con el i.a. Imidacloprid, la mayoría de estos para aplicaciones foliares, con una tasa de aplicación cercana a los $140 \mathrm{~g}$ i.a/ha. La tasa de aplicación alta permitida en Colombia y la comercialización y venta de productos con concentraciones altas, hacen de éste un i.a. de alto riesgo para $B$. atratus; y a esto se le puede sumar la persistencia media reportada para esta molécula, tanto para los tejidos de las plantas como en el suelo (20 y 100 días respectivamente) (Roberts $\&$ Hutson, 1999). Por lo anterior, el uso del Imidacloprid debe considerarse en momentos en los que no haya floración para disminuir el riesgo de contacto directo con los productos y a su vez permitir que la molécula se desintegre dentro y fuera de los tejidos vegetales.

El i.a. Spinosad es considerado altamente tóxico para diferentes especies de abejas (Megachile spp., Osmia spp., Bombus spp.) (Mayes, Thompson, Husband, \& Miles, 2003), sin embargo en el presente estudio no fue posible establecer un límite de respuesta cercano al $100 \%$ para la prueba de toxicidad por exposición tópica. Los límites de respuesta se realizaron con la dosis recomendada por el fabricante y el doble de ésta (0.036 y 0.06 g i.a./L).
Besard et al. (2010) encontraron que la concentración letal media (CL50) tópica para $B$. terrestris fue de $0.12 \mathrm{~g}$ i.a./L, la cual corresponde al doble de la máxima evaluada en éste trabajo. Por lo tanto, la toxicidad letal media aguda por exposición tópica para las obreras de $B$. atratus debe ser evaluada con concentraciones mayores a las utilizadas en éste estudio. Para los resultados obtenidos por exposición oral, el i.a. Spinosad es altamente tóxico para B. atratus (DL50< $1 \mu \mathrm{g}$ i.a./abeja) con una sensibilidad mayor (CL50 $=0.037 \mathrm{~g}$ i.a./L) que la reportada para B. terrestris (CL50 $=0.09 \mathrm{~g}$ i.a./L) (Biondi et al., 2012). Sin embargo, éste i.a. se puede considerar de bajo riesgo debido a la baja tasa de aplicación recomendada (HQ), por su rápida degradación (1-3 días), y su baja actividad sistémica y traslaminar (Roberts \& Hutson, 1999). Morandin et al. (2005) en $B$. impatiens y Biondi et al. (2012) en A. mellifera han demostrado que tasas menores a 100 g i.a./ha. no afectan ni la supervivencia ni el comportamiento de las obreras de estas dos especies. En este sentido, las aplicaciones se podrían realizar en momentos en los que las flores no se encuentren abiertas o no haya una alta actividad de abejas, como puede ser hacia el final de la tarde.

En Colombia, el único producto comercial a base del i.a. Thiocyclam hidrogenoxalato fue registrado en 1995, y a la fecha no existe ningún reporte sobre su efecto en insectos benéficos, a pesar de su amplio uso en el país (Bojacá et al., 2012). Los resultados del presente trabajo constituyen las primeras evidencias de su efecto en abejas. A partir del DL50 por exposición tópica y oral muestran que el i.a. Thiocyclam hidrogenoxalato es altamente tóxico para las obreras de B. atratus (DL50 < $1 \mu \mathrm{g}$ i.a./abeja). Su uso en la tasa de aplicación recomendada para Colombia presenta un riesgo moderado por exposición tópica y un riesgo alto por exposición oral para las obreras de $B$. atratus. Sin embargo, la rápida degradación de la molécula tanto en el suelo como en la planta (1 día) (Roberts \& Hutson, 1999) y su baja actividad sistémica hacen de éste un producto relativamente seguro para $B$. atratus, 
por lo que se podría sugerir hacer uso en horas del día en las que no se observe actividad forrajera de las abejas, momento que dependerá de la zona en donde ocurran los cultivos y el momento de la apertura de la flor.

El panorama sobre la responsabilidad de los efectos de los insecticidas en organismos no blanco, incluyendo las abejas, recae no solamente en las compañías agroquímicas o en los entes locales de regulación gubernamental, sino también en los agricultores, quienes deben hacer uso responsable de los productos agroquímicos, respetando las dosis, tasas de aplicación, tiempo residual, periodo de carencia, entre otros aspectos. En éste sentido, es necesario generar conciencia y sobre todo sensibilidad por parte de las entidades de investigación agrícola gubernamentales o privadas, y de los entes que regulan el registro, venta y uso de estos productos, en el tema de la importancia y necesidad de conservación de las abejas para los agroecosistemas y el bienestar el sector.

\section{RESUMEN}

Los efectos de los insecticidas sobre las abejas han cobrado gran atención a nivel mundial, sin embargo, son pocos los estudios sobre el efecto de estos agroquímicos en abejas Neotropicales. Bombus atratus es una especie neotropical, distribuida ampliamente en los Andes y es considerado un polinizador importante de ecosistemas y agroecosistemas altoandinos. Sin embargo, al igual que muchas especies silvestres, se desconoce el efecto de los insecticidas en $B$. atratus. Teniendo en cuenta lo anterior, el presente trabajo determinó la dosis letal media aguda (DL50) por exposición tópica y oral de las formulaciones comerciales de los insecticidas con los ingredientes activos Imidacloprid, Spinosad y Thiocyclam hidrogenoxalato, ampliamente utilizados para el control de plagas de cultivos importantes en Colombia. Las pruebas DL50 se realizaron a partir de modificaciones de los lineamientos establecidos por la EPPO y OEDE para estas pruebas en Apis mellifera. Se evaluaron 5 dosis para cada insecticida y exposición. Se evaluaron 25 obreras de tamaño medio en cada dosis por duplicado. La mortalidad se registró a las 24, 48 y 72 horas después del experimento. Los datos fueron analizados con el modelo de regresión Probit. Para el Imidacloprid la DL50 tópica y oral fue de $0.048 \mu \mathrm{g} / \mathrm{abeja} \mathrm{y}$ $0.010 \mu \mathrm{g} / \mathrm{abeja}$ respectivamente. Para el Thiocyclam hidrogenoxalato la DL50 tópica y oral fue de $0.244 \mu \mathrm{g} / \mathrm{abeja} \mathrm{y}$ de $0.056 \mu \mathrm{g} /$ abeja respectivamente. Para el Spinosad, la DL50 por exposición oral correspondió a $0.28 \mu \mathrm{g} / \mathrm{abeja}$.
No fue posible establecer la DL50 por exposición tópica. A partir del cálculo del Cociente de Riesgo (HQ) e Índice de Toxicidad Relativa, los tres ingredientes activos son altamente tóxicos. Se analiza y discute el riesgo debido al uso de los productos evaluados a partir de la naturaleza química de los insecticidas.

Palabras clave: dosis letal media (DL50), toxicidad, polinizadores, abejorros.

\section{REFERENCIAS}

Abrahamovich, A., Diaz, N., \& Morrone, J. (2004). Distributional patterns of the Neotropical and Andean species of the genus Bombus (Hymenoptera: Apidae). Acta Zoológica Mexicana, 20(1), 99-117.

Aldana, J., Cure, J. R., Almanza, M. T., Vecil, D., \& Rodríguez, D. (2007). Efecto de Bombus atratus (HYMENOPTERA: APIDAE) sobre la productividad de tomate (Lycopersicon esculentum Mill) bajo invernadero en la Sabana de Bogotá, Colombia. Agronomía Colombiana, 25(1), 62-72.

Almanza, M. (2007). Management of Bombus atratus bumblebees to pollinate lulo (Solanum quiotense $\mathrm{L})$, a native fruit from the Andes of Colombia. ZEF. Ecology and Development Series, 50.

Belloti, A. C., Cardona, C., \& Lapoite, S. L. (1990). Trends in pesticide use in Colombia and Brazil. Journal of Agricultural Entomology, 7(3), 191-201.

Besard, L., Mommaerts, V., Abdu-alla, G., \& Smagghe, G. (2011) Lethal and sublethal side effects assessment supports a more bening profile of spinetoram compared with spinosad in bumble bee Bombus terrestris. Pest Management Science, 67(5), 541-547.

Biesmeijer, J. C., Roberts, P. M., Reemer, M., Ohlemüller, R., Edwards, M., Peeters, T., Schaffers, A. P., Potts, S. G., Kleukers, R., Thomas, C., D., Settele, J., \& Kunin, W. E. (2006). Parallel declines in pollinators and insect-pollinated plants in Britain and Netherlands. Science, 313, 351-354.

Biondi, A., Mommaerts, V., Smagghe, G., Viñuela, E., Zappala, L., \& Desneux, N. (2012). The non-target impact of spinosyns on benefical arthropods. Pest Management Science, 68, 1523-1536

Blacquière, T., Smagghe, G., van Gestel, C., \& Mommaerts, V. (2012). Neonicotinoids in bees: a review of concentrations, side effects and risk assessment. Ecotoxicology, 21, 973-992.

Bojaca, C. R., Gil, R., Casilimas, H., \& Schrevens, E. (2012). Modelling the environmental impact of pesticides sprayed on greenhouses tomatoes. A regional case study in Colombia. Acta Horticulturae, 957, 61-68. 
Colla, S., \& Packer, L. (2008). Evidence for decline in eastern North American bumble bees (Hymenoptera: Apidae), with special focus on Bombus affinis Creason. Biodiversity and Conservation, 17, 1379-1391.

Cruz, P., Almanza, M. T., \& Cure, J. R. (2007). Logros y perspectivas de la cría de abejorros del género Bombus en Colombia. Revista de la Facultad de Ciencias Básicas y Aplicadas, 3(1), 49-60.

Dar, S., Khan, Z., \& Abass, A. (2010). Pollinator decline: a major issue in crop production. Research Journal of Agricultural Sciences, 1(4), 491-493.

Desneux, N., Decourtye, A., \& Delpuech, J. M. (2007). The subletal effects of pesticides on beneficial arthropods. Annual Review of Entomology, 52, 81-106.

Freitas, B., Imperatriz-Fonseca, V. L., Medina, L. M., Kleinert, A. M., Galetto, L., Nates-Parra, G., \& QuezadaEuán, J. (2009). Diversity threats and conservation of native bees in the tropics. Apidologie, 40, 332-346.

Gómez, H. (1999). Algunos métodos estadísticos para el estudio de poblaciones de organismos. Colombia: Facultad de Ciencias Agrícolas, Universidad Nacional de Colombia.

Gordon, F., Rizzardi, M., Vinson, S. B., \& Griswold, T. (2009). Decline in bee diversity and abundance from 1972-2004 on a flowering leguminous tree, Andira inermis in Costa Rica at the interface of disturbed dry forest and the urban environment. Journal of the Kansas Entomolgical Society, 82(1), 1-20.

Greenleaf, S., \& Kremen, C. (2006). Wild bee species increase tomato production and respond differently to surrounding land use in northern California. Biological Conservation, 133, 81-87.

Klein, A. M., Vaissiere, B. E., Cane, J., Steffan-Dewenter, I., Cunningham, S., Kremen, C., \& Tscharntke, T. (2009). Importance of pollinators in changing landscapes for world crops. Proceedings of the Royal Society of London, 274, 303-313.

Marletto, F., Patetta, A., \& Manino, A. (2003). Laboratory assessment of pesticide toxicity to bumblebees. Bulletin of Insectology, 56(1): 155-158.

Marrs, T., \& Ballantyne, B. (2004). Pesticide toxicology and international regulation. England: John Willey \& Sons, Ltd.

Mayes, M. A., Thompson, G. D., Husband, B., \& Miles, M. M. (2003). Spinosad toxicity to pollinators and associated risk. Reviews of Environmental Contamination and Toxicology, 179, 37-71.
Mineau, P., Harding, K. M., Whiteside, M., Fletcher, M. R., Garthwaite, D., \& Knopper, I. D. (2008). Using reports of bee mortality in the field to calibrate laboratory-derived test pesticide risk indices. Environmental Entomology, 3(2), 546-554.

Morandin, L., Winston, M. L., Franklin, M. T., \& Abbott, V. A. (2005). Lethal and sub-lethal effects of spinosad on bumble bee (Bombus impatiens Cresson). Pest Management Science, 61, 619-625.

Nates-Parra, G., \& González, V. H. (2000). Las abejas silvestres de Colombia: Por qué y cómo conservarlas. Acta Biologica Colombiana, 5(1), 5-37.

OECD. (1998a). Guidelines for the testing of chemicals number 213. Honeybees, acute oral toxicity test. OECD. Paris: Environmental health and safety division.

OECD. (1998b). Guidelines for the testing of chemicals number 213, Honeybees, acute contact toxicity test. OECD. Paris: Environmental health and safety division.

Ospina, R., Lievano, A., \& Nates-Parra, G. (1987). Patrón de Coloración del Abejorro Social Bombus atratus, Franklin en Cundinamarca, Colombia. Una Población Diferenciada. Revista de Biología Tropical, $35(2), 317-324$.

Pacateque, J., Cruz, P., Aguilar, M., \& Cure, J. R. (2012). Efecto de la alimentación vía bolsillo en etapas tempranas de desarrollo de Bombus atratus (Hymenoptera: Apidae). Revista Colombiana de Entomología, 38(2), 343-346.

Pinheiro, J., \& Freitas, B. (2010). Efeitos letais dos pesticidas agrícolas sobre polinizadores e perspectivas de manejo para os agroecossistemas brasileiros. Oecologia Asutralis, 14(1), 266-281.

Potts, S. G., Biesmeijer, J. C., Kremen, C., Neumann, P., Schweiger, O., \& Kunin, W. (2010). Global pollinator declines trends impacts and drivers. Trends in Ecology \& Evolution, 25(6), 345-353.

Riaño, D., Veloza, M., Cure, J. R., \& Almanza, M. T. (2014). Desarrollo de dos colonias de Bombus atratus (Hymenoptera: Apidae) mantenidas bajo dos modos de alimentación. Revista de la Facultad de Ciencias Básicas y Aplicadas, 10(2), 132-141.

Roberts, T., \& Hutson D. (1999). Metabolic pathways of agrochemicals. Part two. The Royal Society of Chemistry.

Robertson, J. L., Russell, R. M., Preisler, H. K., \& Savin, N. E. (2007). Bioassays with arthropods. CRS press, Taylor \& Francis Group. 
Rundlöf, M., Nilsson, H., \& Smith, H. (2008). Interacting effects of farming practice and landscape context on bublebees. Biological Conservation, 141, 417-426.

Sánchez-Bayo, F. (2012). Insecticides Mode of Action in Relation to Their Toxicity to Non-Target Organisms. Journal of Environment Analytic Toxicology, S4:002 doi:10.4172/2161-0525.S4-002

Scott-Dupree, C. D., Conroy, L., \& Harris, C. R. (2009). Impact of currently used or potentially useful insecticides for canola agroecosystems on Bombus impatiens (Hymenoptera: Apidae), Megachile rotundata (Hymenoptera: Megachilidae) and Osmia lignaria (Hymenoptera: Megachilidae). Journal of Economic Entomology, 102(1), 177-182. van der Steen, J. (2001). Review of the methods to determine the hazard and toxicity of pesticides on bumblebees. Apidologie, 32, 399-406.

Thompson, H., \& Hunt, L. (1999). Extrapolating from honey bees to bumblebees in pesticide risk assessment. Ecotoxicology, 8(3), 147-166.

Thompson, H. (2001). Assessing the exposure and toxicity of pesticides to bumblebees (Bombus spp). Apidologie, 32, 305-321.

USDA. (2009). Technical Group Report on Honeybee Toxicity Testing. July 8-9. 
